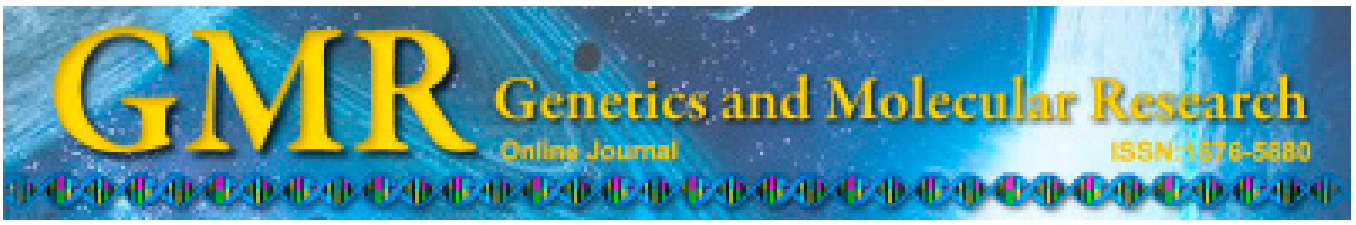

\title{
Bovine yolk sac: from morphology to metabolomic and proteomic profiles
}

\author{
A.C. Galdos-Riveros ${ }^{1}$, P.O. Favaron ${ }^{1}$, S.E.A.L. Will ${ }^{1}$, M.A. Miglino ${ }^{1}$ and \\ D.A. Maria ${ }^{2}$ \\ ${ }^{1}$ Departamento de Cirurgia, \\ Faculdade de Medicina Veterinária e Zootecnia da Universidade de São Paulo, \\ São Paulo, SP, Brasil \\ ${ }^{2}$ Laboratório de Bioquímica e Biofísica, Instituto Butantan, São Paulo, SP, \\ Brasil \\ Corresponding author: P.O. Favaron \\ E-mail: phelipe.favaron@yahoo.com.br
}

Genet. Mol. Res. 14 (2): 6223-6238 (2015)

Received August 25, 2014

Accepted January 19, 2015

Published June 9, 2015

DOI http://dx.doi.org/10.4238/2015.June.9.8

ABSTRACT. In several species, placentation involves the presence
of two different membranes responsible for maternal-fetal exchanges:
the yolk sac and the chorioallantoic placenta. The yolk sac plays
important roles in embryonic survival, mainly during the early stages
of gestation. In bovine, it is a transitional membrane that is present
until day 50-70 of pregnancy. Herein, we evaluated the morphological
and molecular aspects of the yolk sac of bovine embryos during 24 to
52 days of gestation. A total of 69 embryos were allocated into three
groups according to the crown-rump length and estimated ages. Yolk
sac samples were then subjected to morphological and molecular
analysis using mass spectrometry and nuclear magnetic resonance
techniques. In contrast to alanine, which was observed only in Group
I, during all gestational stages, we identified important metabolites
such as aspartate, taurine, glycerophosphocholine, creatinine, creatine,
hydrouracil, glutamate, glutamine, lactate, lysine, valine, myo-inositol,
cadaverine, and choline. In addition, 314 random sequences of proteins 
were identified in the bovine yolk sac, and 47 of these were considered to be specific. Changes in alpha-fetoprotein and carcinoembryonic antigen concentrations during gestation were also evaluated. In conclusion, the majority of these proteins are related to the development of secondary metabolites that are involved in the activation of other proteins and metabolites, and in signaling pathways that are responsible for maternalfetal exchanges, activation of programmed cell death mechanisms, and cellular differentiation, and also in proteins that are responsible for the yolk sac involution that is required to establish chorioallantoic placentation.

Key words: Apoptosis; Metabolic pathways; Proteins; Metabolites; Fetal membranes

\section{INTRODUCTION}

Recently, with the advancement of science, new techniques related to molecular biology have enabled us to identify certain structures and their roles and relationships with the environment in which they operate. In this sense, metabolomics and proteomics have been employed in various areas of science with various purposes. However, these are mainly used to isolate and characterize metabolites in biological samples under normal and experimental conditions (Weckwerth and Morgenthal, 2005). In pharmaceuticals, especially in toxicology and drug development, metabolomics have been used to identify biomarkers of toxicity or disease to understand the mechanisms of action and the selection of effective and safe drugs (Robertson, 2005; Weckwerth and Morgenthal, 2005).

Embryonic development is complex and influenced by many factors, being entirely dependent on an adequate balance between proliferation, differentiation, and cell death. Most cellular functions are coordinated by chemical principles at a molecular level, so that the imbalance of simple compounds in the body can profoundly affect homeostasis. Recent studies have shown that metabolomic changes are associated with the onset and progression of several disease as well as aging (Nicholson, 2006; Chen and Qiu, 2011). In this sense, the yolk sac is a single membrane in charge of nutrition and maintenance of the embryo, especially in early pregnancy, which is marked by intense division and transformation that lead to correct organogenesis. Evolutionarily, the yolk sac is the only embryonic attachment present in all vertebrate species and is also responsible for the production of biomolecules essential for embryonic development, including trophic functions for embryo survival (Wolf et al., 2003). Although structurally diverse among different species, particularly in ruminants, the yolk sac is a large, elongated, and vascularized membrane, which is surrounded by extraembryonic cavity (Assis-Neto et al., 2010). In bovine, the yolk sac develops until the 30th day of gestation as a vascularized membrane (Miglino et al., 2006) and remains present until the 50th day of gestation (Assis-Neto et al., 2010).

Recently, it was reported that changes in the morphogenesis of the yolk sac directly affect the development of other organs and systems, whose functions are vital to the development of the embryo, including bovine generated from artificial insemination, in vitro fertilization and nuclear transfer (Alberto et al., 2012). Their functions are complex in mammals: hematopoiesis and development of the first blood vessels of the embryo, transfer of nutrients, immunoglobulins and gases from the maternal circulation, as well as vitamins and amino 
acids, and is also the site of migration of primordial germ cells (Lloyd et al., 1998; Katayama and Kayano, 1999).

In many mammalian species, the yolk sac produces and transports proteins that are required for embryonic development (Wolf et al., 2003; Galdos-Riveros et al., 2010). It also participates in the exchange of metabolites (Docherty et al., 1996) that are involved in the regulation of biochemical reactions that link different pathways, constitute a cellular network, and are involved in metabolic signaling pathways that regulate growth, differentiation, and cell death (Nielsen et al., 2004).

Given its importance, understanding the functional mechanisms of the yolk sac during early pregnancy, especially in the period of greatest susceptibility to embryonic losses in bovine (23-52 days of gestation, during which the membrane is present), making use of innovative metabolomic and proteomic techniques, outlines the objectives of this study, which may provide valuable information to increase reproductive output and production of these animals, including from an economical point of view.

\section{MATERIAL AND METHODS}

\section{Sample collection}

Altogether, 54 bovine embryos at different gestational ages were collected in slaughterhouses: Barra Mansa (Ribeirão Preto, SP, Brazil), Frigonossa (Poços de Caldas, MG, Brazil), and Cedar Valley (Inhumas, GO, Brazil). Initially, a longitudinal incision was made in the gravid horn, exposing the extra-embryonic membranes. Then, an incision was made in chorioallantoid membrane to access the yolk sac, which was removed and stored in different fixatives (formaldehyde 10\%, Karnowisky, and liquid nitrogen), depending on the technique being used. The procedures for collecting and processing of the yolk sac followed the rules established by the Brazilian College of Animal Experimentation (COBEA). The gestational ages of embryos were estimated according to the crown-rump measurement, which is the distance from the upper end of the nuchal crest to the last sacral vertebra (Evans and Sack, 1973). For this, the embryos were measured with a digital caliper rule (MITUTOYO ${ }^{\circledR}, 500-144 \mathrm{~B}$ ) with divisions in millimeters. Then, the embryos were dissected and photo-documented with a digital camera (Nikon ${ }^{\circledR}$, Model D40).

\section{Light microscopy}

The yolk sac samples were placed in a $10 \%$ formaldehyde solution and transported to the Laboratory of Histology and Embryology, Faculty of Veterinary Medicine and Animal Science, University of São Paulo (USP-FMVZ). After 24-h of fixation, the samples were dehydrated in increasing ethanol concentrations (70-100\%) and deparaffinized in xylene, followed by embedding in paraplast. Blocks were cut into $4-\mu \mathrm{m}$ thick sections in and automatic microtome (LEICA). The slides were stained by hematoxylin and eosin and photo-documented under a light microscope (Olympus BX-50 ${ }^{\circledR}$ ).

\section{Transmission electron microscopy}

After 48-h of fixation in Karnowisky solution, yolk sac samples were washed in phos- 
phate buffer and post-fixed in osmium tetroxide for $2 \mathrm{~h}$. Afterwards, they were washed in buffer, dehydrated in increasing concentrations of ethanol (70-100\%), followed by washing in propylene oxide for $30 \mathrm{~min}$. The fragments were stirred in a 1:1 mixture of propylene oxide and resin (Spurr's kit) for $4 \mathrm{~h}$, and then in pure resin for $12 \mathrm{~h}$. The blocks were cut into $0.4-\mu \mathrm{m}$ thick slices, in an automatic ultramicrotome (ULTRACUT-R Leica Microsystems), and were stained in a $1 \%$ solution of hot toluidine blue. Next, $0.07-\mu \mathrm{m}$ ultrathin sections were obtained that contrasted with saturated uranyl acetate $2 \%$, and by lead citrate $0.5 \%$, each one for 10 min. Analysis and electron micrographs were taken on a transmission electron microscope (Morgagni 268D Mega View III camera ${ }^{\circledR}$, Philips).

\section{Mass spectrometry: nanoUPLC tandem nanoESI-MSE}

To analyze a greater number of proteins, the technology MudPit (Multidimensional Protein Identification Technology) MSE was used in tandem Thompson Laboratory of the Institute of Chemistry, University of Campinas, SP, Brazil.

\section{Sample preparation}

To the pool of 15 yolk sac samples $200 \mu \mathrm{L}$ solution containing $50 \mathrm{mM}$ Tris- $\mathrm{HCl}, \mathrm{pH}$ 8.8, $1.5 \mathrm{mM} \mathrm{KCl}, 0.1 \%(\mathrm{~m} / \mathrm{v})$ SDS and $10 \mathrm{mM}$ DTT was added and was homogenized for 3 min to permit protein extraction. Then, $50 \mu \mathrm{L}$ protein sample was precipitated using $250 \mu \mathrm{L}$ $10 \%(\mathrm{~m} / \mathrm{v})$ trichloroacetic acid solution prepared in acetone at $-20^{\circ} \mathrm{C}$. The solution was stored for $2 \mathrm{~h}$ and centrifuged at $4^{\circ} \mathrm{C}$, for $5 \mathrm{~min}$, at 13,000 $\mathrm{g}$ in an ultracentrifuge (Eppendorf). The formed pellet was dissolved in $200 \mu \mathrm{L}$ phosphate buffer, $\mathrm{pH}$ 7.2. A volume containing $50 \mu \mathrm{g}$ was purified and proteins were concentrated in a centrifugal filter Microcon $0.5 \mathrm{~mL}$ (Amicon Bioseparation, Millipore) to a final concentration of $50 \mu \mathrm{g}$ total proteins in a final volume of $50 \mu \mathrm{L}$.

The process of tryptic digestion was initiated by denaturing the protein with $0.2 \%$ $(\mathrm{w} / \mathrm{v})$ surfactant RapiGest (Waters) for $15 \mathrm{~min}$ at $80^{\circ} \mathrm{C}$. The proteins were reduced using 10$\mathrm{mM}$ DTT (prepared in $50 \mathrm{mM} \mathrm{NH}_{4} \mathrm{HCO}_{3}$ ) for $30 \mathrm{~min}$ at $60^{\circ} \mathrm{C}$. The samples were cooled to room temperature and $10 \mathrm{mM}$ iodoacetamide was added for proteins alkylation for $30 \mathrm{~min}$ in the dark. Then, for the enzymatic digestion of proteins, $10 \mu \mathrm{L}$ trypsin solution in the ratio 1:100 (trypsin/protein) was added. This solution was incubated at $37^{\circ} \mathrm{C}$ overnight. After digestion, $10 \mu \mathrm{L} \mathrm{5 \%}$ trifluoroacetic acid was added at $37^{\circ} \mathrm{C}$ for $90 \mathrm{~min}$ to hydrolyze the surfactant RapiGest ${ }^{\circledR}$. Finally, $5 \mu \mathrm{L}$ internal standard $(25 \mathrm{fmol} / \mu \mathrm{L}$ alcohol dehydrogenase I) was added (SwissProt accession No. P00330).

\section{NanoUPLC tandem nanoESI-MSE}

A total of $250 \mathrm{ng}$ total protein was used to perform this technique. The nanoACQUITY UPLC system utilized a reverse phase gradient from 5-40\% (v/v) acetonitrile $[0.1 \%$ $(\mathrm{v} / \mathrm{v})$ formic acid] with a flow of $600 \mathrm{~nL} / \mathrm{min}$ for $1.5 \mathrm{~h}$ and two types of columns (column C18 BEH $1.7 \mathrm{~mm}, 75 \mathrm{~mm}$ x $15 \mathrm{~cm}$ combined with an SCX column $5 \mathrm{~mm}, 180 \mathrm{~mm}$ x $23 \mathrm{~mm}$ ). For all analyses, the mass spectrometer was operated in "W" mode with a typical resolving power of at least 20,000. The analyses were performed using electrospray ionization in the positive ion mode ESI $(+)$ and a NanoLockSpray source. The lock mass channel was loaded with 
sample every $30 \mathrm{~s}$. Calibration of the mass spectrometer was performed with GFP [(GLU1)Fibrinopeptide human B] $(100 \mathrm{fmol} / \mathrm{mL})$, also provided by the source NanoLockSpray. The doubly charged ion $[(\mathrm{M}+2 \mathrm{H}) 2+]$ was used for single-point calibration (Lteff) initial, and the fragments MS/MS of GFP were used as a final calibration instrument. The MSE part was held in the mass spectrometer, model Synapt HDMS G1 ${ }^{\circledR}$ (Waters), which was set to switch between low-power MS (3eV) and elevated collision energies (MS $\left.{ }^{\mathrm{E}} 12-40 \mathrm{eV}\right)$ applied to the trap 'T-wave' CID (collision-induced dissociation) cell with argon gas. The transfer collision cell was set to $1 \mathrm{eV}$, using a scan time of $1 \mathrm{~s}$, under both low and high energy. After the analyzer flight time (time of flight) spectra of $\mathrm{m} / \mathrm{z} 50-3000$ were collected. The protein identification and quantitative data were generated using algorithms (Silva et al., 2005) and by searching database-specific species (Kramer-Albers et al., 2007). The database used was randomized "on-the fly" during research for generation of false proteins in order to identify the level of false positives, fixed at a maximum of $1 \%$. To process the spectra and search the database, we used the ProteinLynxGlobalServer v.2.4 (PLGs) server licensed ExpressionE v.2.4. The database UniProtKB/Swiss-Prot 57.1 and UniProtKB/TrEMBL Release 40.1 were used and the search conditions were based on the taxonomy of Bos taurus. The proteins were identified by PLGs arranged in a list that corresponds to a single protein, either as the result of the spectrum by the database, and a logarithmic relationship between the different groups. Only proteins with scores greater than 50\% and confidence scores greater than $99 \%$ were considered, and when the same protein was identified by different fragments of MS/MS, only the one with the highest score was considered (Chambery et al., 2009; Li et al., 2009).

\section{Nuclear magnetic resonance ${ }^{1} \mathrm{H}-\mathrm{NMR}$}

Samples of yolk sac preserved in liquid nitrogen were processed for analysis of the metabolome at the Laboratory of Analytical Chemistry, State University of Campinas, SP, Brazil. Experiments ${ }^{1} \mathrm{H}$, uni- and bi-dimensional were acquired on a Bruker Advance $\mathrm{III}^{\circledR}$ spectrophotometer, operating at $400.13 \mathrm{MHz}$ for ${ }^{1} \mathrm{H}$ equipped with a probe under highresolution magic angle spinning (HRMAS) of 4-mm triple resonance (HCP).

\section{Acquisition of spectra}

Experiments of suppression of dual-signal solvent and water via continuous wave and pulsed field gradients were used in addition to 1D sequences using adiabatic methods in their pulse sequences ZGF2PR and CPMG. Two samples of yolk sac of the same gestational age were placed on the rotor and evaluated on a Bruker AdvanceIII ${ }^{\circledR}$ spectrophotometer for $30 \mathrm{~min}$. The NMR-HRMAS allowed the direct analysis of small amounts of tissue (maximum $20 \mathrm{mg}$ ), while keeping this material intact, without the need to treat the sample. By methods of acquiring unidimensional 1D, ${ }^{1} \mathrm{H}$ spectra were initiated and carried out to identify the structures of endogenous metabolites present in sub-millimolar ( $\mathrm{ppm}$ ) concentrations, which are involved in different biochemical pathways. To discriminate the different stages of the yolk sac, variations between samples and direct observations of the spectra were investigated. 1D saturation sequences, T2 filter, and ${ }^{1} \mathrm{H}$ spectra were used. After acquisition, the spectral data were processed using the TopSpin ${ }^{\circledR}$ program, which initiated the identification and characterization of signals by searching the databases and the scientific literature. 


\section{Detection of concentrations of alpha-fetoprotein (AFP) and carcinoembryonic antigen (CEA)}

Yolk sac samples preserved in liquid nitrogen were, after thawing, macerated and subjected to cell lysis by the addition of lysis buffer according to the method described by Galdos-Riveros et al. (2010). To determine the AFP and CEA, commercial kits for chemiluminescence immunoassay (LIAISON ${ }^{\circledR}$, DiaSorin S.p.A) were used. For quantification of CEA, $50 \mu \mathrm{L}$ sample, $270 \mu \mathrm{L}$ buffer $\mathrm{A}$, and $20-\mu \mathrm{L}$ coated magnetic particles were placed into each well and were incubated for $10 \mathrm{~min}$ followed by a wash cycle. Next, $100 \mu \mathrm{L}$ conjugated antibody was added for 10 min followed by a wash cycle. Finally, a $3 \mathrm{~s}$ measurement was carried out and was standardized using a reference standard MRC 73/601. The upper limit of the assay range was considered to be $1000 \mathrm{ng} / \mathrm{mL}$. For quantification of AFP, $25 \mu \mathrm{L}$ sample, $200 \mu \mathrm{L}$ buffer $\mathrm{A}$, and $20-\mu \mathrm{L}$ coated magnetic particles were distributed in each well over 10 min. Next, $200 \mu \mathrm{L}$ conjugated antibody was added for $10 \mathrm{~min}$, followed by a wash cycle. The acquisition was made by measuring for $3 \mathrm{~s}$ and using a standardized reference MRC 72/225. The upper limit of the assay was considered to be $1000 \mathrm{IU} / \mathrm{mL}$.

\section{RESULTS}

For clarity, the results were organized by morphological description of the embryo and yolk sac, and by the metabolomic analysis of the protein profile. The embryos were organized into three different groups according to their estimated ages, which ranged from 23 to 52 days of gestation (Table 1).

$\begin{aligned} & \text { Table 1. Material collected and values for fetal length (Crown-rump - CR) and estimated gestational age } \\
& \text { (according to Evans and Sack, 1973) of bovine embryos. }\end{aligned}$
\begin{tabular}{lcrc}
\hline Group & Number of samples & CR (mm) & Gestational age (days) \\
\hline I & 25 & $5.11-14.90$ & $23-32$ \\
II & 15 & $16.20-24.80$ & $33-42$ \\
III & 14 & $28.15-45.00$ & $43-52$ \\
\hline
\end{tabular}

\section{Morphological analysis of the yolk sac of bovine embryos}

The yolk sac of bovine embryos is located in the ventral portion, presenting itself as a membrane composed of two ends. These extremities in the early stages of the embryonic period (Group I) are quite elongated (Figure 1A). By analyzing the sequence of events that are part of yolk sac development, we observed that at 25 days of gestation (Group I) the embryo is C-shaped, consisting of a pedicle embryonic, neural tube, somites, heart protrusion, mandibular arch, cranial and caudal neuropores, and stomodeum. At 29 days of gestation, the formation of thoracic limbs and the pharyngeal arches began, and were easily identified. The brain region, somites, and heart area were distinguishable. The yolk sac was near the amniotic membrane surrounding the embryo.

In situ staining of yolk sac was yellow with copious blood vessels. In the later stages of embryonic development (Group II), the folding and shortening of the ends toward the umbilical cord occurred, characterizing the process of regression of the yolk sac (Figure 1B and C). 
At the 37th day of gestation (Group II), the embryo showed formation of fore and hindlimbs, retinal pigmentation, anterior region of the brain, cervical curvature, spinal cord, umbilical cord, and oral cavity. The yolk sac is juxtaposed to the amnion, similar to a "dry leaf" when stretched.

Between the 42nd and 48th days of gestation (Group III), the amniotic membrane becomes vascularized, with increased amniotic fluid. The yolk sac is in a process of regression, being juxtaposed to the amnion. The pelvic and thoracic limbs with ends shaped "paddle", pigmented eye, region of the brain, tail and nose region were observed. At 48 days of gestation (Group III), the bifurcation of limbs was observed.

Histologically, a gradual increase in vascularity of the yolk sac was observed in Group I. The abundant blood vessels were present in the yolk sac mesenchyme region (Figure 1D). In the yolk sac of bovine embryos, endodermal cells covered the cavity of the yolk sac and the mesothelium formed an extra-coelomic cavity. Both showed a simple epithelium throughout the embryonic period analyzed, with cuboid endodermis cells and pavement mesothelial cells (Figure 1D). In Group I (28-32 days of gestation), numerous vascular islets were identified along the vitelline membrane, which were full of hemangioblasts (Figure 1D). These remained present until the 42nd day of gestation (Figure 1E), when a reduction in the diameter and the number of blood vessels (Figure 1F) started, although they continued to be present until the 52 nd day of gestation. The endodermic cells showed an extensive system of rough endoplasmic reticulum, numerous vesicles, mitochondria with varied shapes and sizes and a nucleus located in the basal region of the cells (Figure 1G). In the apical surface of these cells, the epithelium showed projections (microvilli) (Figure 1G). Basal invagination of endodermic cells was also observed in the mesenchyme. The mesothelial cells have glycogen deposited in the cytoplasm with few mitochondria and endoplasmic reticulum. Their nuclei were elongated and these cells contacted the endodermis through a thin mesenchymal layer of connective tissue (Figure 1H). During embryonic development, the Golgi apparatus shifted from the apical to the basal region that is closer to the nucleus, reversing the polarity of the cells (Figure 1H). The large number of secretory vesicles and vacuoles present in the cytoplasm of endodermic cells suggests intense secretory or synthesis activity during all stages studied, although there were fewer microvilli present on the apical surface of endodermic cells in Group II (Figure 1I). There was close contact between the endodermic cells in the early stages analyzed, Groups I and II (Figure 1G), which fell considerably in the later stages, Group III, extensive intercellular spaces and breakdown of intracytoplasmic organelles was noted (Figure 1I).

\section{Analysis of metabolomic profile of bovine yolk sac}

The metabolomic profile of the yolk sac of bovine embryos enabled the identification of the metabolic profile between the different gestational periods (Groups I-III). In the 2DNMR spectrum (Figure 2A) the two most significant metabolites can be observed: sn-glycero3-phosphocholine and lactate, which were confirmed by the ${ }^{1} \mathrm{H}-\mathrm{NMR}$ spectrum (Figure 2B), as shown for Group I. The sample period corresponds to the gestational ages in which the yolk sac is more active and is morphologically more developed (Figure 2C). Fifteen metabolites, which are involved in embryonic metabolic pathways, were present in the three groups analyzed: aspartate (3.92 ppm chemical shift - CS), taurine (3.44 ppm CS), sn-glycero-3-phosphocholine (3.15 ppm CS), creatinine (2.95 ppm CS), creatine (3.96 ppm CS), 5,6-dihydrouracil (2.59 ppm CS), glutamate (2.25 ppm CS), glutamine (2.12 ppm CS), alanine - present 
only in Group I (17.3 ppm CS), lactate (1.25 ppm CS), lysine (3.81 ppm CS), valine 0.99 ppm CS), myo-inositol (3.54 ppm CS), cadaverine (1.41 ppm CS) and choline (3.12 ppm CS). The metabolites showed differences in the intensity and chemical shift in Groups I (Figure 3A), II (Figure 3B), and III (Figure 3C). It was noted when comparing the stages of 26 and 41 days, that there is an increase in choline, creatinine, hydrouracil, glutamate, and a decrease in taurine, alanine, and valine.

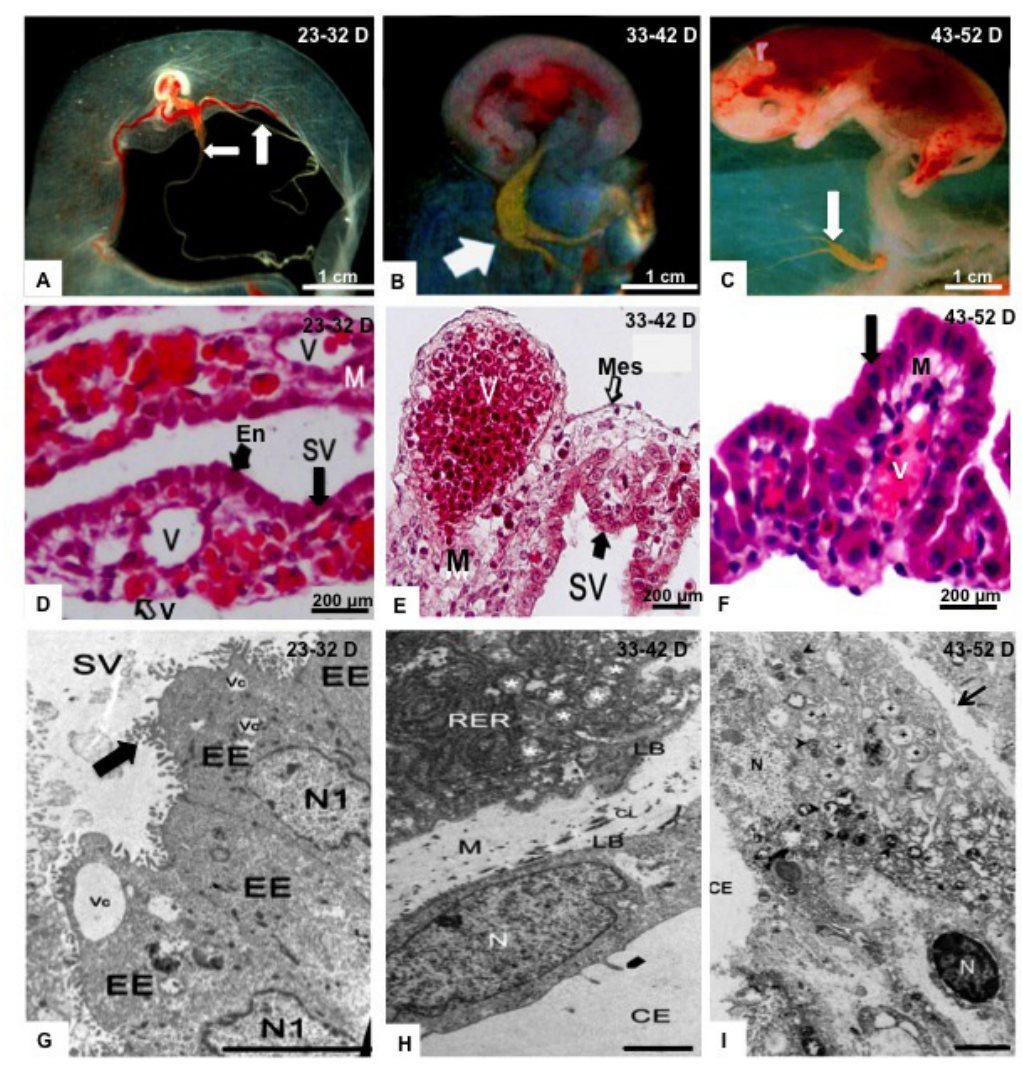

Figure 1. Embryonic development and characteristics of the yolk sac of bovine embryos at different gestational ages. A. B. and C. Representatives of Group I (23-32 days of gestation), II (33-42 days of gestation), and III (4352 days of gestation), respectively, showing major changes in the developing embryo and in the yolk sac (arrows), especially with regard to the involution of the yolk sac. D. E. and F. Light microscopy. Stained using hematoxylin and eosin. Note the intense vascularization (V) in the mesenchyme region (M) of the yolk sac (SV) in Group I, as well as the cubic shape of the endodermal (En) cells. Group II, notice the formation of vascular islets (V) into the mesenchyme (M) of the yolk sac (SV). Note the mesothelium (Mes). In Group III (F), note the decrease in vascularization $(\mathrm{V})$, reduction of the mesenchyme (M) and the prismatic shape of the endodermal cells (arrow). G. H. and I. Transmission electron microscopy of Groups I, II, and III. Note the surface of endodermal cells (EE) covered with microvilli (arrow). Numerous vacuoles (Vc) are present in the apical region of the cytoplasm, while the nucleus $(\mathrm{N})$ is positioned basally. Observe that the intimate contact between the endodermal cells is maintained. In Group II (33-42 days), rough endoplasmic reticulum (RER) and mitochondria (*) are present near the basal lamina (LB). Note the relationship between the mesenchyme and mesothelium, this last represented by the nucleus $(\mathrm{N})$. The yolk sac cells of Group III (I) have characteristic apoptotic with spaces between cells (arrow) disconnecting the perinuclear membrane $(\mathrm{N})$ and disarrangement of intracellular organelles $(+)$. 


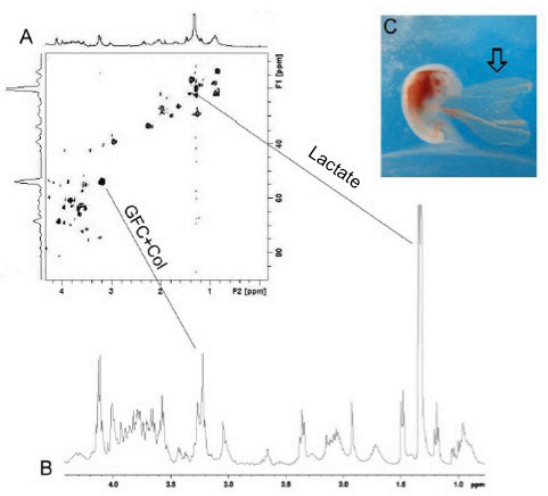

Figure 2. Graphic representation of the metabolome of bovine embryo yolk sac in Group I. A. 2D- ${ }^{1} \mathrm{H}-\mathrm{NMR}$ spectrum. B. 'H-NMR spectrum. C. Characteristic of the bovine embryo yolk sac (arrow) in Group I. For both groups analyzed sn-glycero-3-phosphocholine (CGC) and lactate were the most significant metabolites.

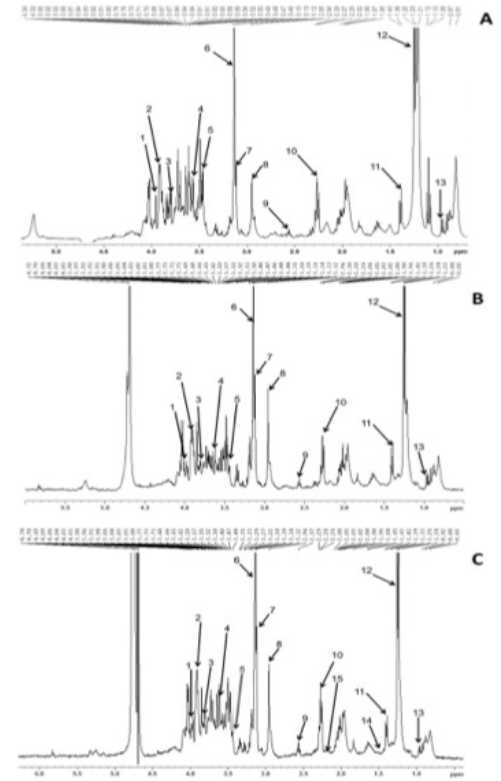

Figura 3. ${ }^{1} \mathrm{H}-\mathrm{NMR}$ spectrum (intensity $\mathrm{x}$ chemical shift in $\mathrm{ppm}$ ) for samples of bovine yolk sac at different gestational ages. A. Group I, B. Group II, and C. Group III. Altogether, 15 metabolites were identified: (1) creatine (chemical shift $3.96 \mathrm{ppm}$ ), (2) aspartate (chemical shift $3.92 \mathrm{ppm}$ ), (3) lysine (chemical shift $3.81 \mathrm{ppm}$ ), (4) myo-inositol (chemical shift $3.54 \mathrm{ppm}$ ), (5) taurine (chemical shift $3.44 \mathrm{ppm}$ ), (6) sn-glycero- 3-phosphocholine (chemical shift $3.15 \mathrm{ppm}$ ), (7) choline (chemical shift $3.12 \mathrm{ppm}$ ), (8) creatinine (chemical shift $2.95 \mathrm{ppm}$ ), (9) 5,6-dihydrouracil (chemical shift $2.59 \mathrm{ppm}$ ), (10) glutamate (chemical shift $2.25 \mathrm{ppm}$ ), (11) cadaverine (chemical shift $3.17 \mathrm{ppm}$ ), (12) lactate (chemical shift $1.25 \mathrm{ppm}$ ), (13) valine (chemical shift $0.99 \mathrm{ppm}$ ), (14) alanine (chemical shift $1.5 \mathrm{ppm}$ ), and (15) glutamine (chemical shift $2.12 \mathrm{ppm}$ ). With the exception of alanine (14), which was found only in Group I, all other metabolites were present in the three groups evaluated.

\section{Analysis of the proteomic profile of bovine embryo yolk sacs}

Protein analysis of the bovine embryo yolk sac revealed 314 random sequences in pooled samples at all ages analyzed. These 314 sequences correspond to 47 unique proteins (Table 2). 
A.C. Galdos-Riveros et al.

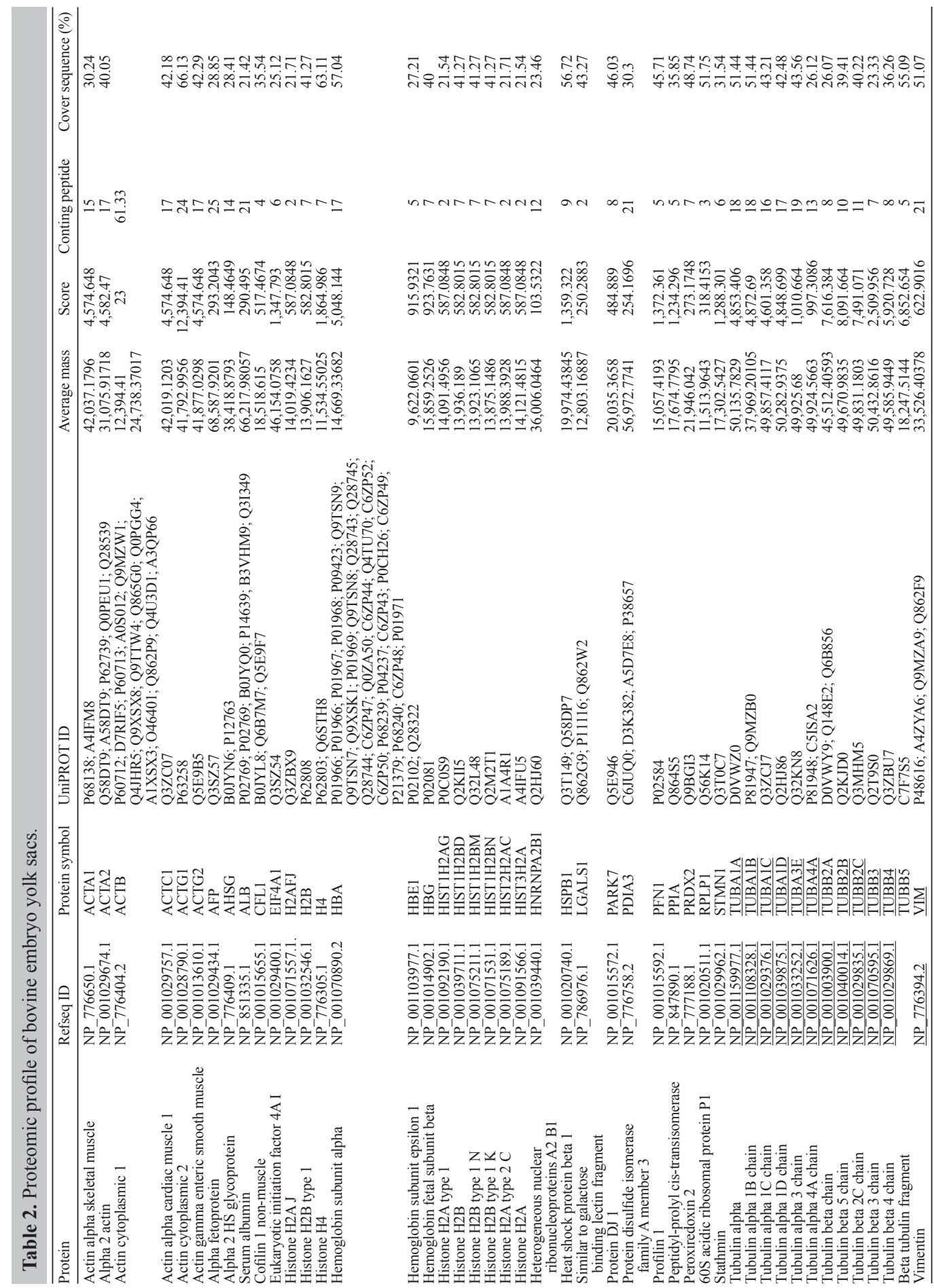




\section{Concentrations of CEA and AFP}

When comparing CEA concentrations in samples of yolk sac at the ages of 26 and 52 days of gestation (early development of yolk sac and involution stage), the concentration of CEA increased with the progression of gestation (Figure 4A). AFP is a glycoprotein produced by the yolk sac, which belongs to the group of oncofetal proteins. The samples from Groups I ( 26 days of gestation) and III (52 days) demonstrated that the AFP concentration undergoes a significant decrease with the progression of gestation (Figure 4B).
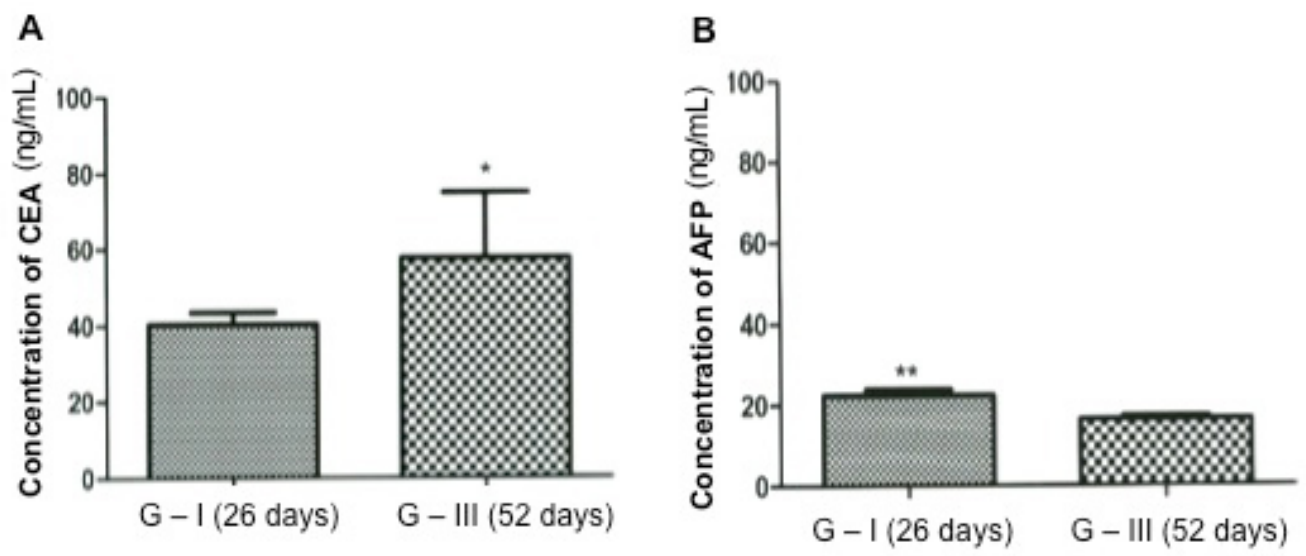

Figure 4. Differences in the concentration of carcinoembryonic antigen (CEA) and alphafetoprotein (AFP) and in the yolk sac of bovine embryos in Groups I (G-I) and III (G-III). A. The graphic shows that the concentration of CEA increases during the progression of gestation. B. The AFP concentration undergoes a significant decrease with the progression of gestation.

\section{DISCUSSION}

\section{General aspects}

Advances in the last two decades in the fields of biochemistry, molecular biology, and cell biology have led to major advances in our understanding of the mechanisms involved in development, cell differentiation, and maturation. Moreover, the search for specific markers for use as diagnostic and prognostic tools coexist with the intense study of the biochemical pathways of cellular metabolism, which may serve as targets for guided or even "customized" therapeutical interventions for each type of organism or cell. The purpose of this study was to establish the known morphological and functional relationships between the yolk sac and embryo. This knowledge will facilitate the understanding and combination of existing embryological studies in future studies, which can assess the real functional impact of the yolk sac during embryonic development through its metabolites and proteins.

Embryonic development marks the beginning of pregnancy in bovine. In this species, the embryonic period extends from fertilization until the 45th day (Evans and Sack, 1973). During this period there are rapid growth and cell differentiation, where tissues, organs, and systems are established and the main external morphological characteristics of the embryonic body are 
recognized. In this study, the embryos were divided into groups according to the crown-rump measurement and external characteristics according to the methodology established by Evans and Sack (1973). Thus, the bovine embryos in Group I had ages ranging from 23 to 32 days of gestation, Group II, of 33-42 days, and Group III, of 43-52 days. The sum of the descriptions of the external characteristics during pregnancy in bovine have been well characterized in the literature for normal pregnancies or in the use of different reproduction techniques such as in vitro fertilization, artificial insemination, and nuclear transfer (Assis-Neto et al., 2010; Alberto et al., 2012).

By analyzing the sequence of events that occur during yolk sac development in bovine embryos, it was observed that at 23 days it was located in the ventral part of the embryo with quite elongated ends. From the 29th day of gestation, the yolk sac decreased in size, particularly at the ends of the membrane. After 37 days, the yolk sac is juxtaposed to the amnion and is less bulky in appearance. Around day 42, their edges folded. Furthermore, we note that the yolk sac of bovine embryos, in the course of embryonic development, changes its anatomical insertion position. First, the yolk sac is located in the ventral region of the embryo formation, which maintains a connection to the primitive gut, which is extremely important for the transfer of nutrients (Mançanares et al., 2013).

In bovine species, the yolk sac is functional for a short period of time (Alberto et al., 2012), but its functions at this stage are of utmost importance for the survival of the embryo. Most importantly, are the absorption and digestion of maternal nutrients, protein synthesis (Galdos-Riveros et al., 2010; Alberto et al., 2012), formation of the vitelline circulation and intravascular secretion of nutrients (Baron, 2003), and some functions performed by the liver, before the fetal liver develops (Gulbis et al., 1998). Furthermore, Alberto et al. (2012) reported that changes in the bovine yolk sac could lead to impairment of correct embryonic development, especially of its organs and structures, as observed in development of the vascular system.

Around days 28-32 of gestation, vascular islets were identified in the yolk sac. It is suggested that central hemangioblasts present in these vessels are related to the development of the first hematopoietic stem cells, while the peripheral hemangioblasts differentiate into angioblasts and precursors of vascular endothelium (Zago and Covas, 2006). Analysis by light and transmission electron microscopy revealed vascular islets in Group II (33-42 days) embryos, mainly in the mesenchymal region of the membrane, as described by Mançanares et al. (2013). In the course of embryonic development, islets cluster and merge with each other, forming endothelial tubes.

Under transmission electron microscopy, the yolk sac showed a single layer of epithelium forming the endoderm with cubic to prismatic cells and abundant cytoplasm. The endodermic cells had microvilli on the surface and secretory vesicles in the apical cytoplasmic region, suggesting high secretory activity by these cells (Alberts et al., 2011). We also note in cuts, yolk sac of early (23-32 days) and medium (33-42 days) gestational age, large amount of rough endoplasmic reticulum, mitochondria, desmosomes, and nuclei with condensed chromatin and, in a lot of cases, more than one nucleolus, showing high proliferative and synthetic activity (Alberts et al., 2011). Moreover, with advancing gestation, the loss of contact between cells, and a reduction in the abundance of microvilli on the apical surface of the endodermic cells were noted. These features contribute to the regression process of the bovine yolk sac.

\section{Metabolomic profile of the yolk sac of bovine embryos}

Embryonic metabolism is important in our understanding of the main factors that al- 
low a pregnancy to reach term. The yolk sac directly contributes to this success early in gestation in bovine, because the membrane is responsible for maternal-fetal exchange, thus being of great interest to study the metabolites present in the yolk sac.

The metabolomic analysis of yolk sac revealed the presence of myo-inositol in all gestational periods. Myo-inositol is an element involved in calcium-dependent signaling; however, there are intrinsic apoptosis pathways linked to quantitative variations of this compound and the formation of intermediate metabolites such as phosphatidylinositol-3-inositol. The derivatives of myo-inositol, such as phosphatidyl inositol-4,5-bisphosphate, play a role in cellular signaling pathways, steroid synthesis, intracellular calcium regulation, and in the maintenance of cellular integrity (Beemster et al., 2002). In vitro experiments suggest that myo-inositol works as a growth factor and its absence can cause changes in the skin, liver, and intestine (Beach and Flick, 1982). There is an interest in myo-inositol in the pathogenesis of reproductive disorders such as infertility, miscarriage, preeclampsia, and congenital malformations such as spina bifida and Down's syndrome (Beemster et al., 2002).

Choline and its derivative glycerophosphocholine were present in yolk sac samples of all three gestational groups, showing that lipid metabolism is an important metabolic pathway for normal development of the embryo. The increase in the ratio of glycerophosphocholine to phosphocholine, obtained by ${ }^{1} \mathrm{H}-\mathrm{NMR}$, correlates with proliferative capacity. Choline is an essential nutrient during fetal development, in the form of phospholipids, such as phosphatidylcholine and sphingomyelin, which are used to synthesize the neurotransmitter acetylcholine. High amounts of choline are required for fetal growth and are transported across the placenta from the maternal circulation (Garner et al., 1993). Choline is a major metabolite that sustains and supports fetal growth. Data suggest that betaine and glycerophosphocholine are involved in the transportation of choline between mother and fetus (Garner et al., 1993). Choline deficiency causes apoptosis; it interrupts the proliferative capacity during organ formation such as liver, kidneys, brain, and the neural tube (Fisher et al., 2002; Craciunescu et al., 2004). Glutamate is another key molecule in cellular metabolism, being the most abundant neurotransmitter in the mammalian central nervous system (Noorlander et al., 2004). In most cell types, this amino acid is involved in metabolic processes such as protein synthesis, energy metabolism, and ammonia fixation. Glutamate metabolism is very important for the growth and development of the fetus. Levels of glutamate in the fetal circulation must be strictly regulated because elevated levels are neurotoxic (Noorlander et al., 2004). Our results showed that glutamate is present throughout the yolk period, suggesting that it has an important role during organogenesis of the bovine embryo. Glutamine is closely related to glutamate, and can be synthesized in various tissues from $\alpha$-ketoglutarate and glutamate. This metabolite plays a number of important physiological roles that include transfer of nitrogen between organs, detoxification of ammonia, and maintenance of acid-base balance during acidosis. It is also a nitrogen precursor for protein synthesis degradation, and an energy substrate for cells of the immune system (Rowbottom et al., 1996).

The yolk sac has several functions during embryo development, for example, it is the place of hematopoiesis initiation (Palis and Yoder, 2001). Our results suggest that the presence of glutamine in the yolk sac of bovine embryos plays an important role in the maintenance of immune cells, especially in the early stages of pregnancy, which coincides with the stage of greatest susceptibility to embryonic loss (Galdos-Riveros et al., 2010).

Taurine is a free amino acid that presents several functions, such as the intracellular antioxidant action, the recovery of toxic intermediates, immunological properties, and regu- 
lation of intracellular calcium. Furthermore, the taurine can be also related to the reduction of the damaging effects of reactive oxygen species formed during in vitro culture (Bidri and Choay, 2003). Knockout mice with taurine deficiency showed a reduction in fertility and loss of vision due to retinal degeneration by apoptotic mechanisms. Taurine is a metabolite that provides a protective function for the embryo, since it inhibits the formation of free radicals. In vivo studies in different species have shown that taurine is essential in several aspects of development in mammals, and that low levels are associated with pathological anomalies and the development of lesions, including cardiomyopathy, retinal degeneration, and growth retardation (Sturman, 1993). Valine was found in the yolk sac of different groups. It is known that valine is related to sickle cell anemia, which is caused by the substitution of glutamic acid for valine in the $\beta$-hemoglobin chain (Falco et al., 1985). This suggests that this metabolite could be used as a potential embryonic biomarker.

Some amino acids are considered to be useful probes for studying neural excitatory neurotransmitters due to their ability to interact specifically with neural systems in mammalian tissues (Hashimoto et al., 1993). Aspartate has the most interaction with metabolic pathways and its presence is vital to the development of the embryo through the yolk sac. According to Fischer et al. (1991), aspartate is present in high concentrations in the cortical area of the brain of embryos during the early stages of pregnancy. In addition, during brain development in rats (Rao et al., 1992), high lysine metabolism was observed, especially in the early stages of development, which gradually decreased during pregnancy. Lysine is involved in the degradation of amino acids. Furthermore, lysine is related to the rate of ovulation and embryonic mortality in pigs (Murgas et al., 1995), as well as a complex mechanism of mitochondrial activation in the liver (Scislowski et al., 1994). Both of these metabolites were identified in the bovine yolk sac during all gestational periods analyzed.

In contrast, creatine was present in low concentrations in the different yolk sac groups. This suggests that the embryo is synthesizing this metabolite, which is involved in metabolic pathways such as the synthesis of urea. The increase of circulating urea could alter the uterine environment and damage the developing embryo. Creatine is a non-essential molecule that is synthesized from arginine, glycine, and methionine primarily in the pancreas and liver (Walker, 1979).

Finally, dihydrouracil was identified in all groups of bovine embryos. This is a metabolite involved in nucleotide metabolism, specifically in the catabolism of pyrimidine-nucleotides, in reactions, or as a precursor of substances of biological interest (Vieira et al., 1998). For this, we infer that the yolk sac retains important metabolic functions even during its involution.

\section{Protein profile of the yolk sac of bovine embryos}

The yolk sac of bovine embryos is an extra-embryonic membrane that contributes significantly to the processes involved in metabolic changes, waste removal, and immune and endocrine transfer during pregnancy (Galdos-Riveros et al., 2010; Alberto et al., 2012). Tubulin or actin cytoskeleton, histone, subunits of hemoglobin, HSP- $\beta 1$, ribosomal protein, the extracellular matrix markers vimentin and annexin 2 and 5 were found in the yolk sac of bovine embryos at all ages analyzed. The annexins are structural proteins that have a calcium activity linked to phospholipids, and are known biomarkers of apoptosis (Reutelingsperger et al., 2002). In the yolk sac of bovine embryos, nine isoforms of actin were found, and were divided into muscle, which is expressed primarily in skeletal muscle, cardiac, and smooth muscle, and cytoplasmic, which are present in all cells. 


\section{CONCLUSION}

Embryonic development is complex, influenced by many factors, and is dependent on an adequate balance between the metabolism that encompasses proliferation, differentiation, and cell death. The yolk sac is an embryonic attachment present in all vertebrate species, and therefore plays key roles during this process. The involution of the yolk sac in bovine embryos, both morphologically and biochemically, determines the end of the first trimester of pregnancy and physiologically allows the embryo to survive to various stimuli. The yolk sac synthesizes and transports important proteins required by the embryo, such as actin or tubulin cytoskeleton, histones, subunits of hemoglobin, HSP- $\beta 1$, ribosomal proteins, and markers of extracellular matrix and vimentin. Moreover, it presents metabolites that are related to the development of the embryo such as alanine, myo-inositol, taurine, choline and glycerophosphocholine, cadaverine, glutamate, glutamine, lactate, hydrouracila, creatine and creatinine, aspartate, and lysine. Metabolomic and proteomic analysis shows that there is a correlation between the presence of metabolites and proteins involved in the production, transportation, and signaling pathways that occur during the yolk stage of bovine embryos.

\section{REFERENCES}

Alberto MLV, Meirelles FV, Perecin F, Ambrósio CE, et al. (2012). Development of bovine embryos derived from reproductive techniques. Reprod. Fertil. Dev. 25: 907-917.

Alberts B, Bray D, Hopkin K, Johnson A, et al. (2011). Fundamentos da Biologia Celular. ARTMED, Porto Alegre.

Assis-Neto AC, Pereira FTV, Santos TC, Ambrósio CE, et al. (2010). Morpho-physical recording of bovine conceptus (Bos indicus) and placenta from Days 20 to 70 of pregnancy. Reprod. Domest. Anim. 45: 760-772.

Baron MH (2003). Embryonic origins of mammalian hematopoiesis. Exp. Hematol. 31: 1160-1169.

Beach DC and Flick PK (1982). Early effect of myo-inositol deficiency on fatty acid synthetic enzymes of rat liver. Biochim. Biophys. Acta 711: 452-459.

Beemster P, Groenen P and Steegers-Theunissen R (2002). Involvement of Inositol in Reproduction. Nutr. Rev. 60: 80-87. Bidri M and Choay P (2003). Taurine: a particular amino acid with multiple functions. Ann. Pharm. Fr. 61: 385-391.

Chambery A, Vissers JPC, Langridge JI, Lonardo E, et al. (2009). Qualitative and quantitative proteomic profiling of crypto (-/-) embryonic stem cells by means of accurate mass LC-MS analysis. J. Proteome Res. 8: 1047-1058.

Chen SZ and Qiu ZG (2011). Combined treatment with GH, insulin, and indomethacin alleviates cancer cachexia in a mouse model. J. Endocrinol. 208: 131-136.

Craciunescu CN, Brown EC, Mar MH, Albright CD, et al. (2004). Folic acid deficiency during late gestation decreases progenitor cell proliferation and increases apoptosis in fetal mouse brain. J. Nutr. 134: 162-166.

Docherty SM, Iies RK, Wathen N and Chard T (1996). The temporary anatomical structures prominent in the first trimester may be fulfilling exchange functions assigned to the placenta in the second and third trimester. Hum. Reprod. 11: $1157-1161$.

Evans HE and Sack WO (1973). Prenatal Development of Domestic and Laboratory Mammals: Growth Curves, External Features and Selected References. Anat. Histol. Embryol. 2: 11-45.

Falco SC, Dumas KS and Livak KJ (1985). Nucleotide sequence of the yeast ILV2 gene which encodes acetolactate synthase. Nucleic Acids Res. 13: 4011-4027.

Fischer GH, D'aniello A, Vetere A, Padula L, et al. (1991). Free D-aspartate and D-alanine in normal and Alzheimer brain. Brain Res. Bull. 26: 983-985.

Fisher MC, Zeisel SH, Mar MH and Sadler TW (2002). Perturbations in choline metabolism cause neural tube defects in mouse embryos in vitro. FASEB J. 16: 619-621.

Galdos-Riveros AC, Toledo-Piza A, Rezende LC, Maria DA, et al. (2010). Proteômica: novas fronteiras na pesquisa clínica. Enciclopédia Biosfera 6: 24.

Garner SC, Chou SC, Mar MH, Coleman RA, et al. (1993). Characterization of choline metabolism and secretion by human placental trophoblasts in culture. Biochim. Biophys. Acta 1168: 358-364.

Gulbis B, Jauniaux E, Cotton F and Stordeur P (1998). Protein and enzyme patterns in the fluid cavities of the first trimester gestational sac: relevance to the absorptive role of secondary yolk sac. Mol. Hum. Reprod. 4: 857-862. 
Hashimoto A, Kumashiro S, Nishikawa T, Oka T, et al. (1993). Embryonic development and postnatal changes in free $\mathrm{d}$-aspartate and d-serine in the human prefrontal cortex. J. Neurochem. 61: 348-351.

Katayama I and Kayano H (1999). Yolk Sac with Blood Island. N. Engl. J. Med. 340: 617-617.

Kramer-Albers EM, Bretz N, Tenzer S, Winterstein C, et al. (2007). Oligodendrocytes secrete exosomes containing major myelin and stress-protective proteins: Trophic support for axons? Proteomics - Clin Appl. 1: 1446-1461.

Li GZ, Vissers JPC, Silva JC, Golick D, et al. (2009). Database searching and accounting of multiplexed precursor and product ion spectra from the data independent analysis of simple and complex peptide mixtures. Proteomics 9: 16961719.

Lloyd JB, Beckman DA and Brent RL (1998). Nutritional role of the visceral yolk sac in organogenesis-stage rat embryos. Reprod. Toxicol. 12: 193-195.

Mançanares CAF, Leiser R, Favaron PO, Carvalho AF, et al. (2013). A morphological analysis of the transition between the embryonic primitive intestine and yolk sac in bovine embryos and fetuses. Microsc. Res. Tech. 76: 756-766.

Miglino MA, Ambrósio CE, Martins DS, Wenceslau CV, et al. (2006). The carnivore pregnancy: The development of the embryo and fetal membranes. Theriogenology 66: 1699-1702.

Murgas LDS, Torres CAA and Donzele JL (1995). Efeito do consumo de energia sobre a idade e peso corporal de marrãs à puberdade. Rev. Soc. Bras. Zoot. 24: 409-416.

Nicholson JK (2006). Global systems biology, personalized medicine and molecular epidemiology. Mol. Syst. Biol. 2: 52.

Nielsen TO, Hsu FD, Jensen K, Cheang M, et al. (2004). Immunohistochemical and clinical characterization of the basallike subtype of invasive breast carcinoma. Clin. Cancer Res. 10: 536-74.

Noorlander CW, De Graan PNE, Nikkels PGJ, Schrama LH, et al. (2004). Distribution of glutamate transporters in the human placenta. Placenta 25: 489-495.

Palis J and Yoder MC (2001). Yolk-sac hematopoiesis: The first blood cells of mouse and man. Exp. Hematol. 29: 927-936.

Rao VV, Pan X and Chang YF (1992). Developmental changes of 1-lysine-ketoglutarate reductase in rat brain and liver. Comp. Biochem. Physiol. B Comp. Biochem. 103: 221-224.

Reutelingsperger CP, Dumont E, Thimister PW, van Genderen H, et al. (2002). Visualization of cell death in vivo with the annexin A5 imaging protocol. J. Immunol. Methods 265: 123-32.

Robertson DG (2005). Metabonomics in Toxicology: A Review. Toxicol. Sci. 85: 809-822.

Rowbottom DG, Keast D and Morton AR (1996). The Emerging Role of Glutamine as an Indicator of Exercise Stress and Overtraining. Sports Med. 21: 80-97.

Scislowski PW, Foster AR and Fuller MF (1994). Regulation of oxidative degradation of L-lysine in rat liver mitochondria. Biochem. J. 300: 887-891.

Silva JC, Denny R, Dorschel CA, Gorenstein M, et al. (2005). Quantitative proteomic analysis by accurate mass retention time pairs. Anal. Chem. 77: 2187-2200.

Sturman JA (1993). Taurine in development. Physiol. Rev. 73: 119-147.

Vieira EC, Gazzinelli G and Mares-Guia M (1998). Bioquímica Celular e Biologia Molecular. Atheneu, Belo Horizonte.

Walker JB (1979). Creatine: biosynthesis, regulation, and function. Adv. Enzymol. Relat. Areas Mol. Biol. 50: 177-242.

Weckwerth W and Morgenthal K (2005). Metabolomics: from pattern recognition to biological interpretation. Drug Discov. Today. 10: 1551-1558.

Wolf E, Arnold GJ, Bauersachs S, Beier HM, et al. (2003). Embryo-Maternal Communication in Bovine - Strategies for Deciphering a Complex Cross-Talk. Reprod. Domest. Anim. 38: 276-289.

Zago MA and Covas DT (2006). Células-tronco: a nova fronteira da medicina. Atheneu, Rio de Janeiro. 